\title{
Clinical and Economic Burden of Pneumococcal Disease Due to Serotypes Contained in Current and Investigational Pneumococcal Conjugate Vaccines in Children Under Five Years of Age
}

\author{
Matt D. Wasserman (D) · Johnna Perdrizet (D) - Lindsay Grant • \\ Kyla Hayford (D) - Shubhra Singh · Paranjoy Saharia · Emily K. Horn (D) • \\ Raymond A. Farkouh
}

Received: August 24, 2021 / Accepted: September 28, 2021 / Published online: October 11, 2021

(C) The Author(s) 2021

\section{ABSTRACT}

Introduction: The widespread implementation of pneumococcal conjugate vaccines (PCVs) has significantly reduced the burden of pneumococcal disease around the world. Although licensed 10-valent (PCV10) and 13-valent (PCV13) vaccines have considerably reduced mortality and morbidity, a sizeable disease burden attributable to serotypes not contained in these PCVs remains. This study aimed to estimate the annual clinical and economic burden of pneumococcal disease attributable to licensed (PCV10 and PCV13) and investigational PCVs, notably 15-valent (PCV15) and

Supplementary Information The online version contains supplementary material available at https:// doi.org/10.1007/s40121-021-00544-1.

M. D. Wasserman · J. Perdrizet · E. K. Horn ·

R. A. Farkouh

Pfizer Inc., Health Economics and Outcomes

Research, New York, USA

L. Grant · K. Hayford

Pfizer Inc., Medical and Scientific Affairs, New York, USA

S. Singh $\cdot$ P. Saharia

IQVIA, Real-World Solutions, Gurgaon, India

M. D. Wasserman $(\bowtie)$

Patient and Health Impact, Global Health

Economics and Outcomes Research, Pfizer Inc., 235

42nd Street, New York, NY 10017, USA

e-mail: matt.wasserman@pfizer.com 20-valent (PCV20) vaccines, in 13 countries in children under 5 years of age.

Methods: A decision-analytic model was created to aggregate total cases [inclusive of invasive pneumococcal disease (IPD), pneumonia, and otitis media $(\mathrm{OM})]$, deaths, and direct costs in each country of interest [stratified by PCV10/ PCV13 countries, depending on national immunization programs (NIPs)] over 1 year, using up to the three most recent years of available serotype coverage data. Data inputs were sourced from local databases, surveillance reports, and published literature.

Results: In 5 PCV10 NIPs (Austria, Finland, Netherlands, New Zealand, Sweden), most remaining PCV20-type disease was due to PCV13-unique serotypes (30-85\%), followed by PCV20-unique (9-50\%), PCV15-unique (4-15\%), and PCV10-unique (2-14\%) serotypes. In 8 PCV13 NIPs (Australia, Canada, France, Germany, Italy, South Korea, Spain, United Kingdom), most remaining PCV20-type disease was caused by PCV20-unique serotypes (16-69\%), followed by PCV13-unique (11-54\%), PCV15-unique (2-33\%), and PCV10unique serotypes (3-19\%). Across all countries, PCV20 serotypes caused 3000 to 345,000 cases of disease and cost between $\$ 1.3$ and $\$ 44.9$ million USD annually with variability driven by population size, NIP status, and epidemiologic inputs. In aggregate, PCV20 serotypes caused $1,234,000$ cases and $\$ 213.5$ million in annual 
direct medical costs in children under 5 years of age.

Conclusion: Despite the success of PCV10 and PCV13 in reducing pneumococcal disease, a substantial clinical and economic burden remains due to serotypes contained in investigational vaccines.

Keywords: Invasive pneumococcal disease; Pneumococcal conjugate vaccine; Burden of disease; Vaccine serotypes

\section{Key Summary Points}

\section{Why carry out this study?}

Currently licensed pneumococcal conjugate vaccines (PCVs) i.e., PCV10 and PCV13 have reduced the burden of pneumococcal disease; however, due to a rise in the prevalence of serotypes not covered by these PCVs, a significant proportion of disease burden remains to be addressed.

This study sought to estimate and compare the clinical and economic burden attributable to the serotypes covered by licensed PCVs, as well as investigational PCVs (PCV15 and PCV20) that are currently in development, in 13 countries with established national immunization programs (NIPs) and robust surveillance data.

\section{What was learned from the study?}

Serotypes not targeted by licensed PCVs are a substantial cause of the residual disease burden in all 13 countries; notably the largest proportion of the residual clinical and economic burden is due to PCV20 serotypes.

Investigational PCVs have the potential to reduce the disease burden in these countries by targeting additional serotypes that are not covered by lowervalent PCVs.

\section{INTRODUCTION}

Streptococcus pneumoniae (S. pneumoniae), the causative pathogen for pneumococcal disease, is one of the leading contributors of vaccine-preventable morbidity and mortality worldwide $[1,2]$. Pneumococcal disease can range from life-threatening invasive pneumococcal disease (IPD), such as meningitis, septicemia, bacteremia, and bacteremic pneumonia (PNE), to mucosal infections, such as otitis media (OM), non-bacteremic PNE, and sinusitis. Notably, children younger than 5 years of age, older adults, and adults with comorbid conditions are most affected by pneumococcal disease [3]. Pneumonia due to $S$. pneumoniae is a significant contributor to childhood morbidity and mortality, and estimates from 2016 indicate that around 45 million episodes of lower respiratory infections and 350,000 deaths are due to pneumococcal PNE in children younger than 5 years of age annually [4].

Pneumococcal disease is caused by 100 different pneumococcal serotypes, but most cases are caused by a subset of pathogenic serotypes [5]. To address the burden of pneumococcal disease in children, pneumococcal conjugate vaccines (PCVs) were developed and originally targeted serotypes most prevalent among IPD (Fig. 1). The 7-valent PCV (PCV7) containing 7 pneumococcal serotypes $(4,6 \mathrm{~B}, 9 \mathrm{~V}, 14,18 \mathrm{C}$, $19 \mathrm{~F}$, and 23F) was the first PCV to be approved in 2000 in the United States, and later in Europe in 2001 [6, 7]. In 2009, a 10-valent PCV (PCV10) containing additional serotypes 1,5 , and $7 \mathrm{~F}$ and a 13-valent PCV (PCV13) containing PCV10 serotypes and additional serotypes 3, 6A, and 19A were introduced, replacing PCV7 in national immunization programs (NIPs) $[6,8]$. As of 2020, PCV10 and PCV13 have been introduced in 160 NIPs around the world [9]. Considering the incremental serotype coverage provided by PCV13, over $80 \%$ of NIPs currently include PCV13 to provide the broadest protection against pneumococcal disease [9].

Over the last 20 years, use of PCV in pediatric NIPs around the world has demonstrated a significant impact in reducing morbidity and mortality associated with pneumococcal disease 


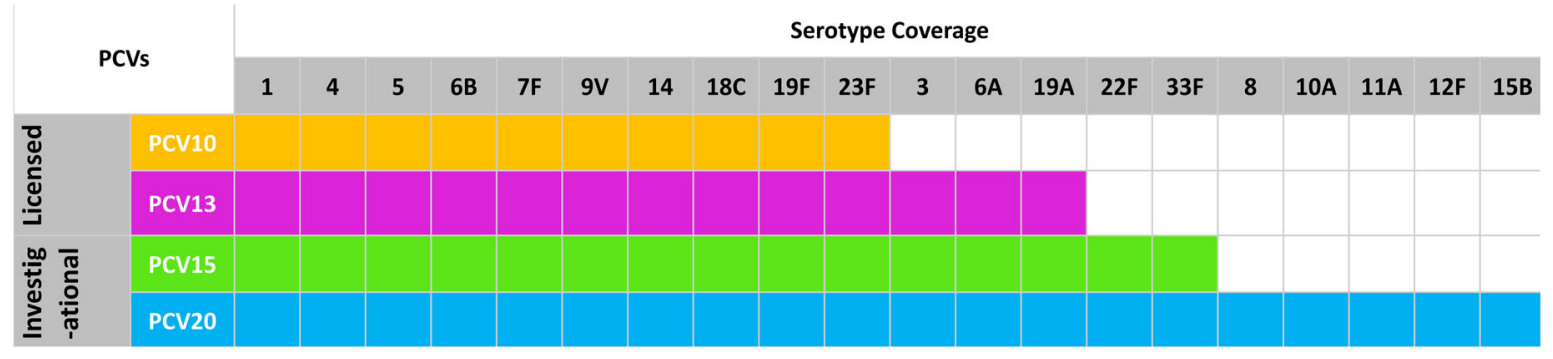

Fig. 1 Serotypes included in current and investigational PCVs. $P C V$ pneumococcal conjugate vaccine

caused by vaccine serotypes in children. In the years following the introduction of PCV7, and again after its subsequent replacement by PCV10/PCV13, there have been marked declines in cases of IPD [10-13], OM [14-17], and PNE [18-21] attributable to vaccine serotypes. Global modeling studies provide further evidence of the impact of PCV programs, estimating that 175 million cases of pneumococcal disease and 625,000 deaths have been prevented by PCV13 in children under 5 years of age worldwide between 2010 and 2019 [1]. Apart from protecting young children against pneumococcal disease, routine pediatric immunization has also provided indirect protection to non-vaccinated populations through a reduction in nasopharyngeal carriage of vaccine serotypes, thereby reducing transmission of disease [19, 22, 23]. As more countries increase PCV uptake, PCV vaccination offers the potential to prevent 54.6 million episodes and 399,000 deaths annually in children under 5 years of age due to pneumococcal disease globally [24].

While PCVs have considerably reduced disease burden of vaccine serotypes, there has been a rise in cases caused by non-vaccine serotypes, a phenomenon termed serotype replacement [25]. This replacement occurs because S. pneumoniae is a commensal bacterium that resides in the nasopharynx of healthy children and currently available PCVs offer protection against nasopharyngeal carriage of covered pneumococcal serotypes. While overall nasopharyngeal carriage has remained constant, the non-vaccine serotypes that have replaced the vaccine serotypes in circulation are generally less pathogenic, therefore overall rates of IPD remain substantially lower than pre-vaccine levels. However, wide variations in the incidence of these emerging serotypes by geography and age have been observed [26-29]. Countries with sustained use of PCV13 in NIPs, where the incidence of serotypes $3,6 \mathrm{~A}$, and 19A have already been reduced as compared to countries with PCV10 NIPs, have observed an increase in the incidence of multiple non-PCV13 serotypes, including but not limited to serotypes 6C, 8, $9 \mathrm{~N}, 10 \mathrm{~A}, 11 \mathrm{~A}, 12 \mathrm{~F}, 15 \mathrm{~A}, 15 \mathrm{~B}, 15 \mathrm{C}, 22 \mathrm{~F}, 23 \mathrm{~B}, 24 \mathrm{~F}$, $33 \mathrm{~F}, 35 \mathrm{~B}$, and 38 [27]. Other global studies have reported on the high disease potential of serotypes not contained in currently licensed PCVs [30-32].

To address the rise in non-vaccine serotypes and increase protection against pneumococcal disease, higher-valent PCVs are in clinical development, with two vaccines currently under regulatory review $^{1}$ : a 15 -valent PCV (PCV15) that includes the PCV13 serotypes plus 22F and 33F and a 20-valent PCV (PCV20) that includes the PCV15 serotypes and 5 additional serotypes (8, 10A, 11A, 12F, and 15B) (Fig. 1). These new PCV formulations have the potential to address an increasing unmet need by providing additional protection against emerging serotypes not covered by currently licensed PCVs. Notably, the serotypes included in investigational PCVs have a propensity for antimicrobial resistance $(11 \mathrm{~A}, 15 \mathrm{~B}, 22 \mathrm{~F}$, and $33 \mathrm{~F})$, are associated with outbreaks ( 8 and $12 \mathrm{~F}$ ), are a common cause of meningitis $(12 \mathrm{~F}, 22 \mathrm{~F}$, and 33F), have a higher case fatality rate (CFR)

\footnotetext{
1 The pneumococcal 20-valent conjugate vaccine PREVNAR $20^{\circledR}$ (Pfizer) and the pneumococcal 15-valent conjugate vaccine VAXNEUVANCE ${ }^{\circledR}$ (Merck) received marketing approval for adults aged 18 years or older in June 2021 and July 2021, respectively [42, 43].
} 
(10A and $11 \mathrm{~A}$ ), and tend to cause more severe disease $(8,10 \mathrm{~A}, 11 \mathrm{~A}, 15 \mathrm{~B}, 22 \mathrm{~F}$, and 33F) compared with other serotypes [33-41]. The aim of this study is to estimate and compare the annual clinical and economic burden of pneumococcal disease outcomes including IPD, inpatient PNE, outpatient PNE, and OM due to serotypes attributable to the licensed (PCV10 and PCV13) and investigational PCVs (PCV15 and PCV20) in 13 countries with established NIPs and robust surveillance data. Specifically, this analysis considers Australia, Austria, Canada, Finland, France, Germany, Italy, Netherlands, New Zealand, South Korea, Spain, Sweden, and United Kingdom (UK), in children under 5 years of age.

\section{METHODS}

\section{Model Description}

A Microsoft Excel-based decision-analytic model was developed to estimate the annual clinical and economic pneumococcal disease burden due to serotypes contained in licensed and investigational PCVs (PCV10, PCV13, PCV15, and PCV20) in children under 5 years of age (Fig. 2). The cohort-based model is based on a cohort of children under 5 years of age and includes age- and country-specific incidence rates of pneumococcal disease outcomes (i.e., IPD, inpatient/outpatient PNE, and OM) which are used to calculate the estimated number of pneumococcal disease cases per year. Disease outcomes are subsequently stratified based on local serotype coverage for each PCV. Thereafter, age- and country-specific associated costs and CFRs are applied to pneumococcal disease outcomes to estimate the direct costs and total deaths associated with each disease outcome by PCV product. Finally, total cases, deaths, and direct costs are aggregated to estimate the annual clinical and economic burden of disease in each country over 1 year. This decision analytic model is informed by previously conducted studies and does not contain any new studies with human participants or animals performed by any of the authors.
Inclusion criteria for countries in this study were the presence of well-established national population-based pneumococcal surveillance systems and public data available after the introduction of PCV10/PCV13, including published evidence of non-invasive pneumococcal disease (non-IPD) incidence and disease-related costs. Following a targeted literature review, Australia, Austria, Canada, Finland, France, Germany, Italy, Netherlands, New Zealand, South Korea, Spain, Sweden, and UK were chosen for this analysis (Table 1).

Austria, Finland, and Netherlands exclusively used PCV10 in their NIP at the time serotype coverage was reported, while Australia, France, Spain, and UK exclusively use PCV13 for routine pediatric vaccination. Austria switched its NIP to PCV13 at the beginning of 2020; however, for the purposes of this analysis, it is assumed to use PCV10, given serotype coverage is available from 2017 to 2019 [44]. Spain, Italy, and Canada have regional immunization programs; however, while all of Spain uses PCV13, one region in Italy and Canada uses PCV10 (Piedmont in Italy and Quebec in Canada), with the remaining regions using PCV13. Quebec switched to PCV10 in 2018, so only 6 months of epidemiologic data for Canada represent any PCV10 use. In Germany and South Korea, both PCV13 and PCV10 are reimbursed; acknowledging this, most infants $(>85 \%)$ are vaccinated with PCV13 [45, 46]. Therefore, Italy, Canada, Germany, and South Korea are categorized as PCV13 NIPs in our study, given that most infants receive PCV13 in these countries. In Sweden, individual counties could choose either PCV10 or PCV13 until 2019, after which PCV10 was selected at the national level as the only PCV [47]. However, the majority of children in Sweden received PCV10 before 2019; therefore, we have categorized it as a PCV10 NIP [48]. Finally, New Zealand has switched its PCV program several times, switching from PCV7 to PCV10 in 2011, then to PCV13 in 2014, and back to PCV10 in 2017. We categorized New Zealand as a PCV10 country and included only 2018 surveillance data post-PCV10 transition. 


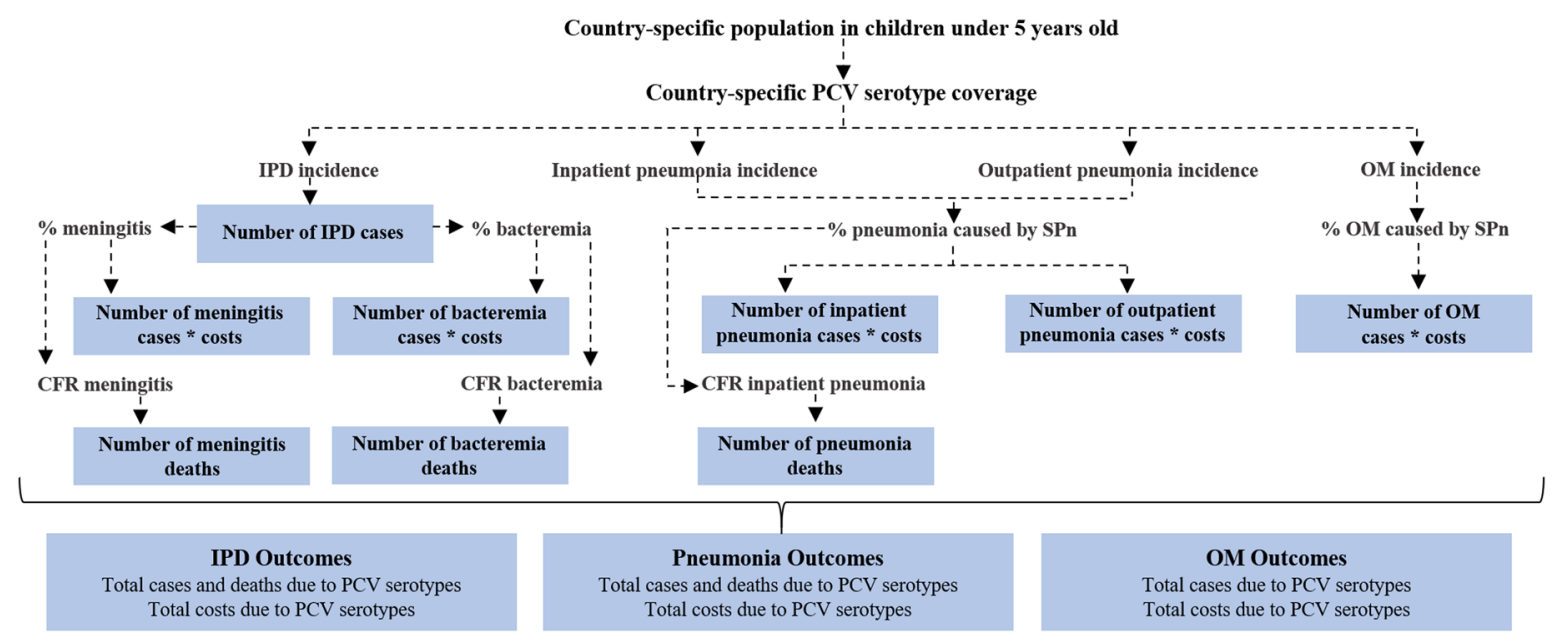

Fig. 2 Derivation of clinical and cost outcomes. CFR case fatality rate, IPD invasive pneumococcal disease, $O M$ otitis media, $P C V$ pneumococcal conjugate vaccine, SPn Streptococcus pneumoniae

\section{Model Inputs}

Input parameters (i.e., population, clinical, and cost data) were derived from published sources available in the public domain or from locally available databases/registries (see Table $\mathrm{S} 1$ in the supplementary material for details). The model inputs derived per country for children under 5 years of age included: (1) Serotype coverage: the proportion of serotypes in each PCV formulation (PCV10, PCV13, PCV15, and PCV20) responsible for cases of IPD; (2) IPD incidence: the total incidence of IPD, and the relative proportion caused by meningitis or bacteremia; (3) non-IPD (OM/PNE) incidence: the incidence of all-cause $\mathrm{OM}$, the incidence of all-cause PNE (inpatient and outpatient), and the proportion of all-cause OM/PNE caused by $S$. pneumoniae; (4) Mortality: the CFR for meningitis, bacteremia, and inpatient PNE; and (5) Direct healthcare costs: the direct healthcare cost per event of meningitis, bacteremia, inpatient PNE, outpatient PNE, and OM.

\section{Serotype Coverage}

Serotype coverage for children under 5 years of age was derived from nationally or regionally representative IPD surveillance systems for each country. Using up to the three most recent years of available data, coverage was calculated for PCV10-, PCV13-, PCV15-, and PCV20-unique serotypes. Only 1 year of data was used in New Zealand and Netherlands to account for the only year of data since switching to a PCV10 NIP and the availability of data by individual serotypes, respectively. In South Korea, 4 years of data were used, given that the passive surveillance system reported aggregated data over the entire period. In the base case analysis, we did not include any potentially cross-reactive serotypes (i.e., 6C or $15 \mathrm{C}$ ); however, this was explored in the sensitivity analysis. These serotype coverage estimates were then applied to IPD incidence to ascertain the nationally representative serotype-specific IPD incidence for each PCV considered in the analysis.

Country-specific serotype coverage for licensed and investigational PCVs is presented in Fig. 3. In Austria, Finland, Netherlands, New Zealand, and Sweden, countries that all used PCV10 in the NIP at the time of data collection, $1-9 \%$ of all cases were caused by PCV10-unique serotypes. Among these countries, disease caused by PCV13-unique serotypes $(3,6 \mathrm{~A}$, and 19A) accounted for most of the remaining disease, ranging from $15-66 \%$, with an additional $3-8 \%$ caused by the PCV15-unique serotypes (22F and 33F), and a further $7-33 \%$ caused by the five additional PCV20-unique serotypes (8, $10 \mathrm{~A}, 11 \mathrm{~A}, 12 \mathrm{~F}$, and 15B). In countries predominantly using PCV13, i.e., Australia, Canada, France, Germany, Italy, South Korea, Spain, and 
Table 1 Overview of pediatric NIPs in selected countries

\begin{tabular}{|c|c|c|c|}
\hline Country & $\begin{array}{l}\text { PCV used during reported surveillance } \\
\text { (dosing schedule) }\end{array}$ & $\begin{array}{l}\text { Year of PCV10/13 } \\
\text { introduction }\end{array}$ & $\begin{array}{l}\text { Years serotype coverage } \\
\text { data reported }\end{array}$ \\
\hline \multicolumn{4}{|l|}{ PCV10 NIPs } \\
\hline Austria $^{a}$ & PCV10 $(2+1)$ & 2012 & $2017-2019$ \\
\hline Finland & PCV10 $(2+1)$ & 2010 & $2017-2019$ \\
\hline Netherlands & PCV10 $(2+1)$ & 2011 & 2019 \\
\hline $\begin{array}{l}\text { New } \\
\text { Zealand }^{\mathrm{b}}\end{array}$ & PCV10 $(3+1)$ & 2011 & 2018 \\
\hline Sweden ${ }^{c}$ & PCV10 $(2+1) / \operatorname{PCV} 13(2+1)$ & 2010 & $2016-2018$ \\
\hline \multicolumn{4}{|l|}{ PCV13 NIPs } \\
\hline Australia $^{\mathrm{d}}$ & $\operatorname{PCV} 13(3+0 / 2+1)$ & 2010 & $2016-2018$ \\
\hline Canada $^{e}$ & $\operatorname{PCV} 13(2+1) / \operatorname{PCV} 10(2+1)$ & 2010 & $2016-2018$ \\
\hline France & PCV13 $(2+1)$ & 2010 & 2013, 2015, 2017 \\
\hline Germany $^{\mathrm{f}}$ & $\operatorname{PCV} 13(2+1) / \operatorname{PCV} 10(2+1)$ & 2009 & July 2015-June 2018 \\
\hline Italy $^{\mathrm{e}}$ & $\operatorname{PCV} 13(2+1) / \operatorname{PCV} 10(2+1)$ & 2012 & $2017-2019$ \\
\hline South Korea ${ }^{\mathrm{g}}$ & PCV13 $(3+1) / \operatorname{PCV} 10(3+1)$ & 2014 & $2014-2018$ \\
\hline Spain & PCV13 $(2+1)$ & 2010 & $2016-2018$ \\
\hline $\begin{array}{l}\text { United } \\
\text { Kingdom }\end{array}$ & PCV13 $(2+1)$ & 2010 & July 2016-June 2017 \\
\hline
\end{tabular}

NIP national immunization program, $P C V$ pneumococcal conjugate vaccine

a At the time of available serotype coverage data, Austria used PCV10 but has since changed to PCV13 as of 2020

b New Zealand switched from PCV7 to PCV10 in 2011, then to PCV13 in 2014, and back to PCV10 in 2017 , which is why only 2018 data was included

c At the time of serotype coverage data availability, Sweden used both PCV10 and PCV13 in different counties, but has exclusively used PCV10 since 2020

d Australia used a $3+0$ schedule and transitioned to a $2+1$ schedule in 2018

e Italy and Canada both have regional PCV10 use in Piedmont and Quebec, respectively

f Both PCV10 and PCV13 are used in Germany, with over 90\% of infants vaccinated with PCV13. Published data for Germany were available for children $<2$ years; this was assumed uniform for all children $<5$ years

g Both PCV10 and PCV13 are used in South Korea, with over 85\% of infants vaccinated with PCV13

h Surveillance is specifically from England and Wales, but this analysis extrapolates to the totality of the population under 5 years of age in the UK. The UK has also switched from $2+1$ to $1+1$ schedule as of 2020

UK, $2-11 \%$ of disease was caused by PCV10unique serotypes, with an incremental $6-33 \%$ of disease caused by PCV13-unique serotypes. Additional PCV15-unique serotypes were responsible for $1-18 \%$ of disease in these countries, while the additional PCV20-unique serotypes were responsible for $10-41 \%$ more disease over PCV15. Across both PCV10 and
PCV13 NIP countries, the cumulative coverage of PCV20 ranged from $46-77 \%$ of all remaining pneumococcal disease burden.

\section{Invasive Pneumococcal Disease}

The proportion of IPD caused by either pneumococcal meningitis or bacteremia was 


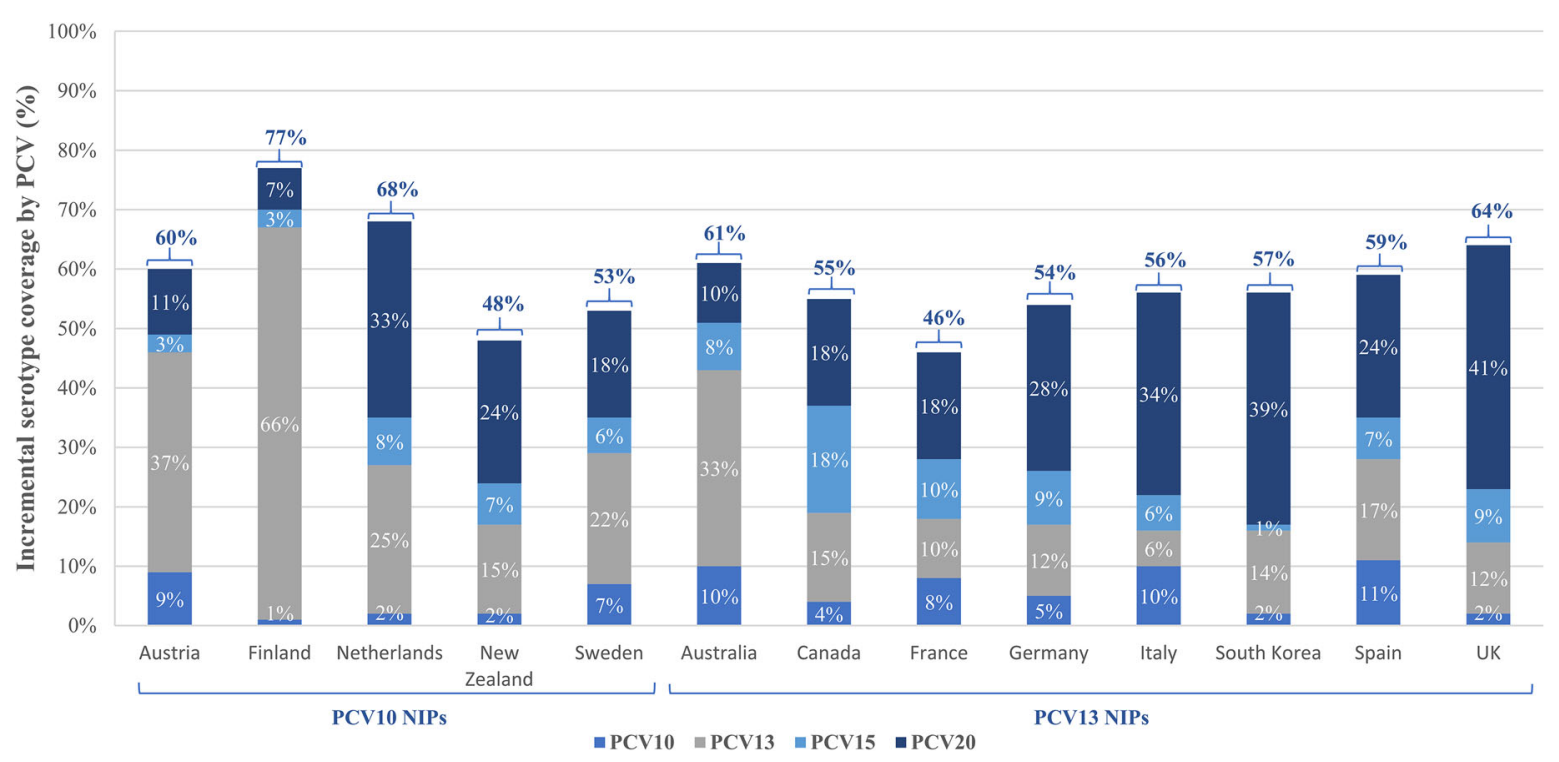

Fig. 3 Incremental serotype coverage by $\mathrm{PCV}$, in children $<5$ years in 13 selected countries. PCV10-serotypes: $1,4,5,6 \mathrm{~B}, 7 \mathrm{~F}, 9 \mathrm{~V}, 14,18 \mathrm{C}, 19 \mathrm{~F}$, and 23F; PCV13serotypes: PCV10 serotypes $+3,6 \mathrm{~A}$, and 19A; PCV15serotypes: PCV13 serotypes $+22 \mathrm{~F}$ and $33 \mathrm{~F} ; P C V 20$ -

calculated to capture differences in CFRs and associated costs per disease event. Due to the lack of available data on the proportion of IPD cases resulting in meningitis or bacteremia in four countries (UK, Finland, Austria, and Netherlands), it was assumed that $76-90 \%$ of IPD cases were pneumococcal bacteremia, and the remaining $10-24 \%$ were pneumococcal meningitis, as per previously published work $[49,50]$. Sequelae resulting from meningitis and bacteremia were not included in our analysis.

\section{Non-Invasive Pneumococcal Disease}

Non-IPD in the model included inpatient PNE, outpatient PNE, and OM. Data on the pneumococcal etiology of non-IPD in children are sparse and inconsistent to fully ascertain vaccine-preventable disease burden. Consequently, we leveraged available data on all-cause PNE and $\mathrm{OM}$ for each country, identified through a targeted literature review, and assumed that a proportion of all-cause disease was caused by $S$. pneumoniae. This proportion ranged from 20-53\% for inpatient and outpatient PNE and $20-38 \%$ for OM among included countries. For countries where no data were available, we serotypes: PCV15 serotypes $+8,10 \mathrm{~A}, 11 \mathrm{~A}, 12 \mathrm{~F}, 15 \mathrm{~B}$. $N I P$ national immunization program, $P C V$ pneumococcal conjugate vaccine, $U K$ United Kingdom

conservatively assumed that $20 \%$ of both allcause PNE and OM disease were attributable to S. pneumoniae [46, 51, 52]. Furthermore, given that the etiology is not known, the serotypes causing non-IPD are also uncertain. Therefore, we assumed that the serotype distribution causing non-IPD disease was equivalent to IPD, consistent with previous economic burden and cost-effectiveness evaluations [46, 52, 53].

\section{Case Fatality Rates}

Bacteremia, meningitis, and inpatient PNE carried a risk of death and their respective countryspecific CFRs were taken from published literature for children under 5 years of age. Outpatient PNE and OM were assumed to have no risk of death.

\section{Economic Inputs}

The study took a healthcare payer perspective and only considered the direct medical acute healthcare costs associated with each respective health state as reported in each country (see Table S1 in the supplementary material for details). We therefore did not include any costs 
of productivity loss due to caregiver time or missed work due to illness. We also did not include long-term costs associated with any potential sequelae of disease following acute conditions such as meningitis, as these were considered rare, and data were inconsistently reported across countries. All local costs were inflated to 2020 values in United States dollars (USD) based on local consumer price index (CPI) values.

\section{Analysis}

Leveraging country-level population data of children under 5 years of age, IPD serotype coverage proportions were extrapolated to calculate the annual number of cases of IPD (bacteremia and meningitis), inpatient and outpatient PNE, and OM, as well as the annual number of deaths and associated costs attributable to each disease outcome. From this extrapolation, the cumulative number of cases, deaths, and direct costs associated with serotypes in each PCV formulation were estimated. The resulting annual vaccine-preventable clinical and economic burden in children under 5 years of age were reported for each PCV by country. Absolute serotype coverage and burden by PCV serotype group are presented in tables, while incremental differences are discussed in the text.

Sensitivity analyses were also undertaken to evaluate the impact of cross-reactive serotypes 6C for PCV13, PCV15, and PCV20 and 15C for PCV20 on the potential preventable disease burden and associated costs (see Tables S2 and S3 in the supplementary material for details). This analysis was conducted because studies have shown that PCV13 may elicit a crossfunctional opsonophagocytic killing response between serotype $6 \mathrm{~A}$, contained in PCV13, and serotype 6C [54]. This has been substantiated with observed reductions in IPD caused by serotype 6C in countries using PCV13. Similarly, it is hypothesized that serotype $15 \mathrm{~B}$ antigen may be cross-reactive with serotype $15 \mathrm{C}$ due to a high degree of genetic homology [55]. Furthermore, in some countries, serotypes $15 \mathrm{~B}$ and $15 \mathrm{C}$ are not differentiated in the laboratory and are reported together as $15 \mathrm{~B} / \mathrm{C}$. For countries reporting serotype data in this manner (i.e., France, Italy, and UK), the percentage of IPD due to $15 \mathrm{~B} / \mathrm{C}$ was consequently excluded in PCV20 base case coverage estimates but was included in sensitivity analyses. We did not include a sensitivity analysis where PCV10 provides cross-protection for serotype 19A, despite PCV10 showing some early cross-reactivity between serotypes 19F and 19A [56]. This hypothesized cross-protection was excluded because countries using PCV10 as part of their NIP have observed a steady rise in cases due to serotype 19A [56, 57].

\section{RESULTS}

\section{Clinical Burden}

Annual pneumococcal disease morbidity and mortality attributable to PCV10, PCV13, PCV15, and PCV20 serotypes were evaluated across the included countries in children under 5 years of age (Table 2). In addition, the estimated incremental proportion of disease caused by PCV serotypes, excluding non-PCV20 serotypes, is captured in Fig. 4.

\section{PCV10 NIP Countries (Austria, Finland, Netherlands, New Zealand, and Sweden)}

In PCV10 NIP countries, the disease burden due to serotypes covered by PCV10 was $2-14 \%$ (398-4170 cases annually) of the remaining PCV20-type disease. In Austria and Finland, an additional $62-85 \%$ of remaining PCV20-type disease $(17,957-21,976$ cases annually) was due to PCV13-unique serotypes; in Netherlands, New Zealand, and Sweden, PCV13-unique serotypes accounted for a comparatively lower proportion of residual disease, i.e., $30-41 \%$ (1303-5509 cases annually). An additional 4-5\% (1162-1374 cases annually) and 9-19\% (2324-5544 cases annually) vaccine-preventable cases in Austria and Finland were attributable to PCV15-unique serotypes and PCV20-unique serotypes, respectively. In contrast, the proportion of remaining PCV20-type disease due to PCV15-unique and PCV20unique serotypes was higher in Netherlands, 


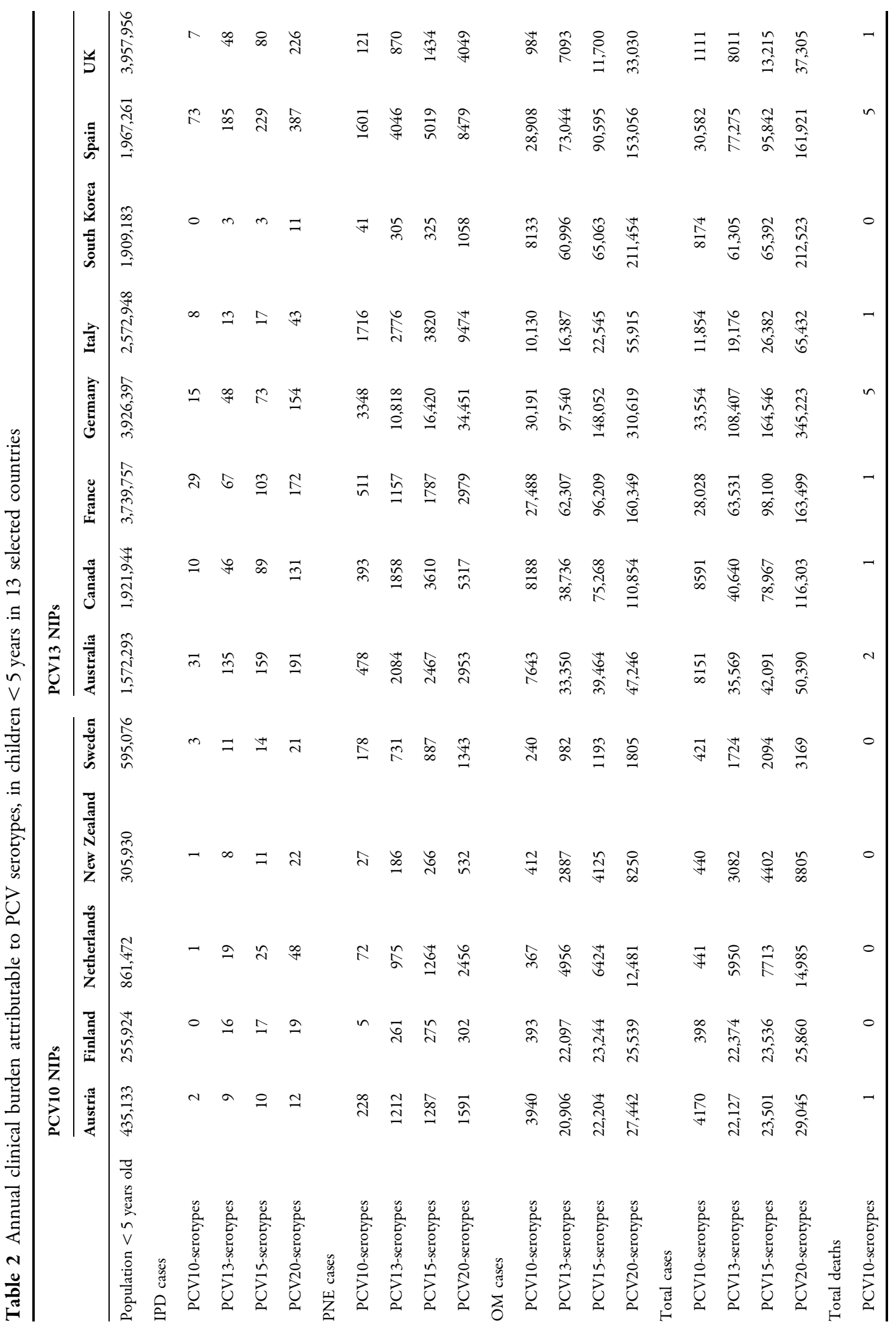




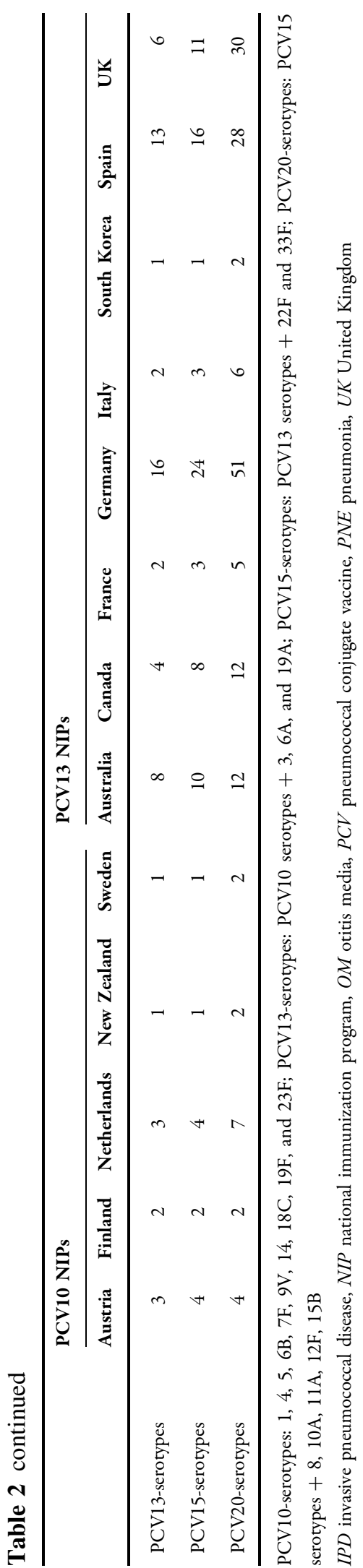

New Zealand, and Sweden, estimated to be $12-15 \%$ (370-1763 cases annually) and $34-50 \%$ (1075-7272 cases annually), respectively. Similar trends were modeled in the 5 countries for all outcomes (IPD cases, PNE cases, OM cases, and total deaths).

PCV13 NIP Countries (Australia, Canada, France, Germany, Italy, South Korea, Spain, and $U K$ )

In the eight countries that predominantly use PCV13 as part of their NIP, the remaining PCV20-type disease burden due to PCV10unique serotypes ranged from 3-19\% (1111-33,554 cases annually). An additional $11-29 \%$ of vaccine-preventable cases (6900-74,853 cases annually) were due to the PCV13-unique serotypes; Australia had a relatively higher distribution at $54 \%(27,418$ cases annually). An additional $2-33 \%$ of vaccinepreventable cases (4087-56,139 cases annually) were attributable to the PCV15-unique serotypes, whereas the most remaining cases i.e., 16-69\% (8299-180,677 cases annually) were due to the additional PCV20-unique serotypes. Similar trends were modeled in the 8 countries for all outcomes (IPD cases, PNE cases, OM cases, and total deaths).

\section{Economic Burden}

Across all countries, similar trends were observed in the proportion of annual economic burden attributable to the incremental PCV serotypes as compared to the presented clinical burden. The remaining direct economic burden due to PCV10-unique serotypes ranged from \$0.06-6.74 million, with an additional economic burden of \$0.40-10.29 million due to PCV13-unique serotypes, and \$0.18-7.30 million due to PCV15-unique serotypes. The PCV20-unique serotypes contributed to a considerably higher proportion of annual economic burden ranging from \$0.37-23.48 million in annual health system costs (Table 3). The annual economic burden due to PCV10-, PCV13-, PCV15-, and PCV20-unique serotypes across all 13 markets was $\$ 25.39$ million, \$62.79 million, \$30.18 million, and \$95.14 million, 


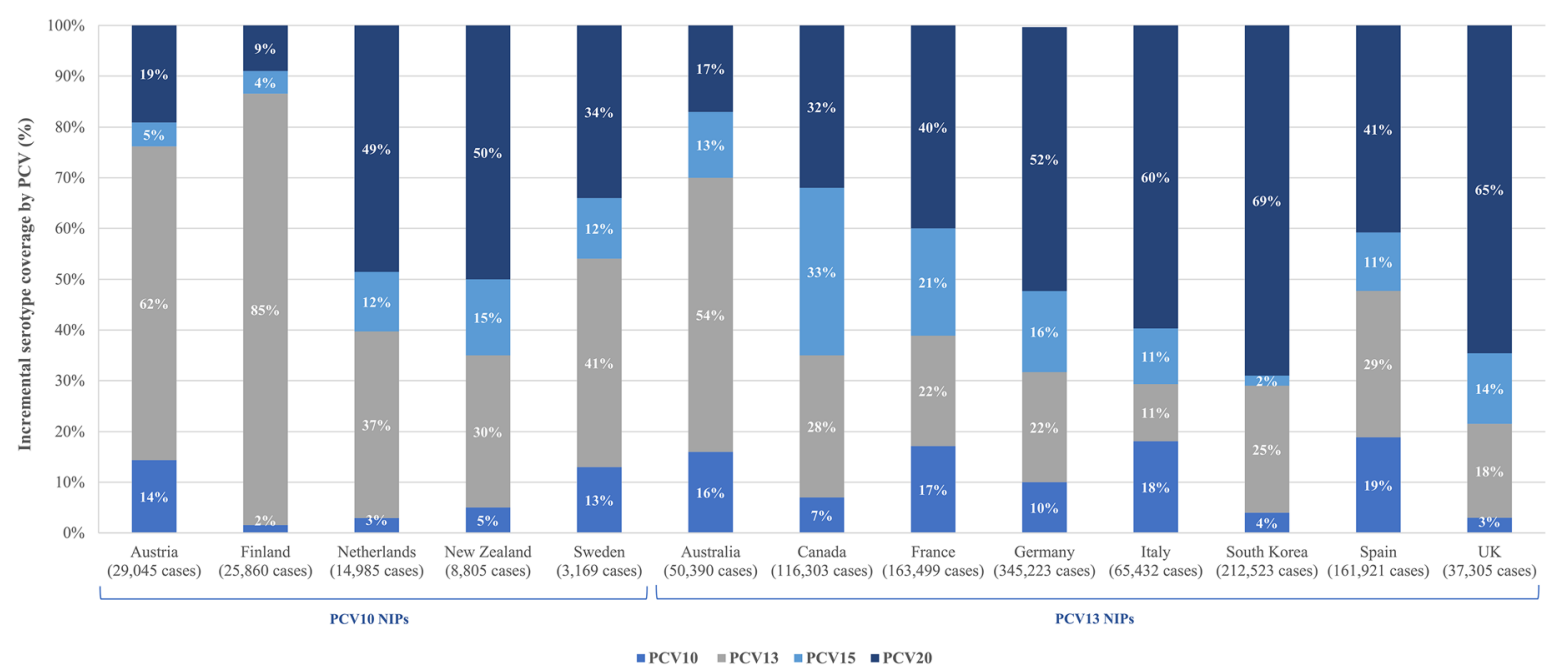

Fig. 4 Proportion of PCV20-type disease burden covered by PCV10, PCV13, PCV15, and PCV20 in children $<5$ years in 13 selected countries. Figure represents the relative PCV serotype coverage (\%) of total pneumococcal disease cases (caused by PCV10, PCV13, PCV15, PCV20

respectively. Across countries and outcomes (i.e., IPD cases, PNE cases, and OM cases), the highest annual economic burden was mostly reported for OM cases due to the high prevalence of $\mathrm{OM}$ as compared to other pneumococcal disease outcomes.

\section{Sensitivity Analysis}

A sensitivity analysis was performed to assess the impact of including cross-reactive serotypes 6C with PCV13, PCV15, and PCV20, as well as 15C with PCV20. The analysis was conducted for Australia, Austria, Canada, Finland, France, Italy, Netherlands, New Zealand, South Korea, Spain, Sweden, and UK, which reported IPD cases caused by either serotypes 6C or 15C (see Tables S2 and S3 in the supplementary material for details). Among these countries, serotype coverage of $6 \mathrm{C}$ and $15 \mathrm{C}$ ranged from $0.3 \%$ to $8.5 \%$ and from $0 \%$ to $9.5 \%$, respectively.

In Austria and Finland, countries with PCV10 NIPs, the remaining PCV20-type disease burden (inclusive of cross-reactive serotypes) due to PCV13-, PCV15-, and PCV20-unique serotypes was $63-86 \%(22,078-23,536$ cases annually), 4\% (1162-1374 cases annually), and serotypes) by country, exclusive of non-PCV20 type disease. NIP national immunization program, $P C V$ pneumococcal conjugate vaccine, $U K$ United Kingdom

8-22\% (2324-7605 cases annually), respectively. In the other PCV10 markets, i.e., Netherlands, New Zealand, and Sweden, the remaining PCV20-type disease burden differed from Austria and Finland, with disease cases due to PCV13-, PCV15-, and PCV20-unique serotypes estimated to be $33-47 \%$ (1814-6390 cases annually), $\quad 10-14 \% \quad(369-1763$ cases annually), and 33-48\% (1263-7272 cases annually), respectively. Compared to the base case, the inclusion of $6 \mathrm{C}$ and $15 \mathrm{C}$ in PCV13 serotype coverage increased the number of cases caused by PCV13-unique serotypes by $7-39 \%$ in the five PCV10 markets, while $17 \%$ and $37 \%$ more cases were caused by PCV20-unique serotypes in Sweden and Austria, respectively.

In the 7 PCV13 NIPs (Australia, Canada, France, Italy, South Korea, Spain, and UK), the remaining PCV20-type disease burden (inclusive of cross-reactive serotypes) attributable to PCV13-unique serotypes was 11-29\% (7777-57,218 cases annually); it was relatively higher in Australia at 52\% $(28,456$ cases annually). An incremental 2-28\% vaccine-preventable cases (4087-38,327 cases annually) were due to PCV15-unique serotypes, and most of the remaining cases, i.e., $21-71 \%$ $(11,412-171,652$ cases annually) were 


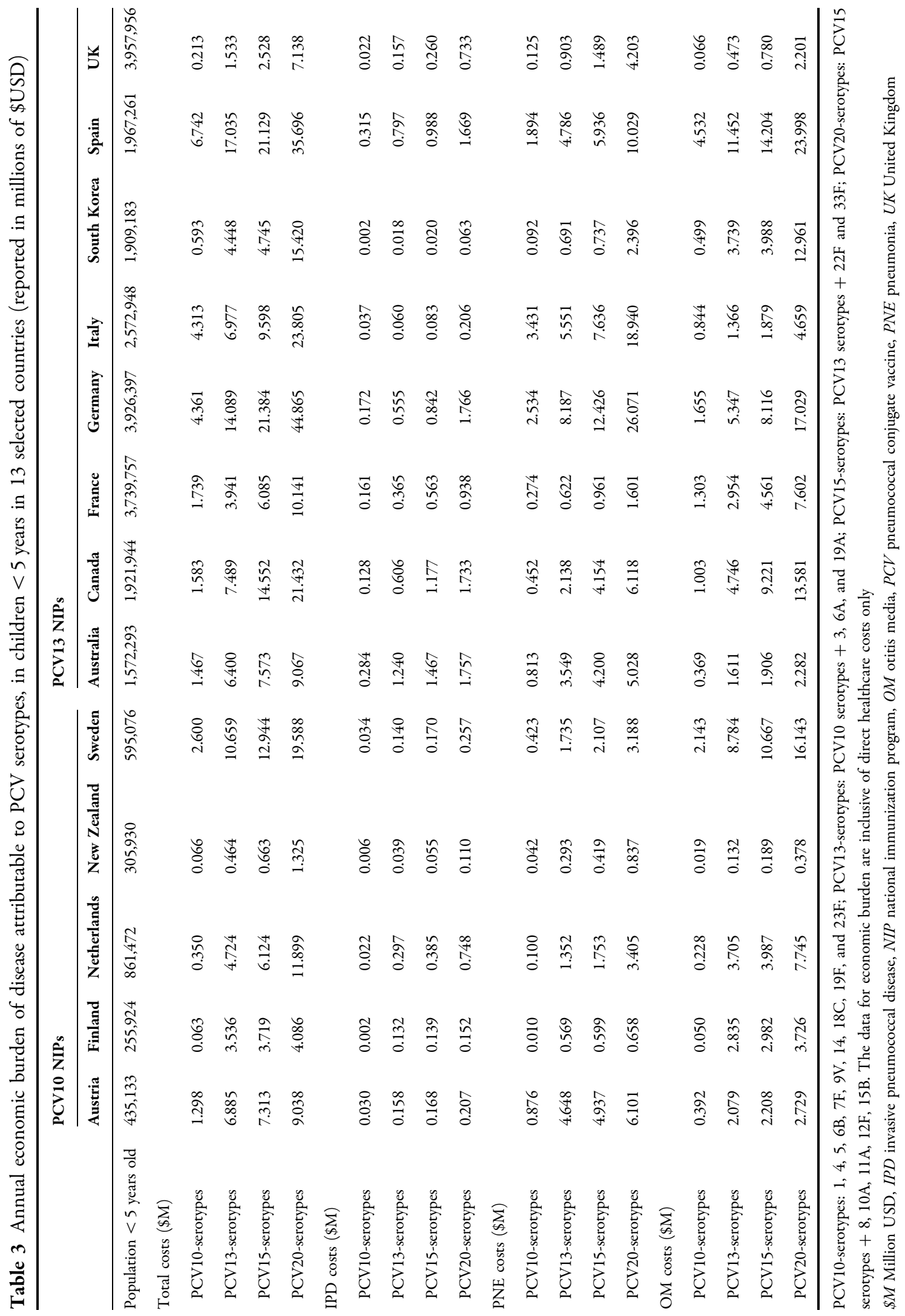


attributable to PCV20-unique serotypes. Across the PCV13 NIP countries, $3-8 \%$ (up to $13 \%$ in UK) additional cases were caused by PCV13unique serotypes and $11-38 \%$ (up to $49-50 \%$ in France and Canada) additional cases were caused by PCV20-unique serotypes with the inclusion of $6 \mathrm{C}$ and $15 \mathrm{C}$ as compared to the base case.

\section{DISCUSSION}

This study presents an analysis of the potential vaccine-preventable pneumococcal clinical and economic disease burden due to serotypes contained in currently licensed and investigational PCVs among children under 5 years of age in countries with PCV10 or PCV13 NIPs. To our knowledge, this is the first multi-country assessment of the vaccine-preventable disease burden of investigational vaccines, as measured through avertable disease cases and deaths and direct health system costs. The results of this analysis demonstrate that there is a substantial unmet need due to serotypes not covered within currently licensed PCVs among countries considered in this study. Notably, between $46 \%$ and $77 \%$ of remaining pneumococcal disease is due to PCV20 serotypes among the 13 countries included in this analysis. This corresponds to approximately 1.23 million cases, 160 deaths, and \$214 million in direct medical costs annually across included countries. These findings align with the reported impact of nonPCV13 serotypes on clinical and economic burden in recent studies; several publications have discussed the remaining disease burden of IPD [58, 59] and OM [60, 61] driven by nonPCV13 serotypes in children under 5 years of age. Extrapolating our findings of clinical and economic disease burden to a global scale, the need for broader PCV serotype coverage is evident.

Of note, the heterogeneity between serotype distributions across countries observed in our study depended primarily on whether PCV10 or PCV13 was used in the NIP. In countries with PCV10 NIPs (i.e., Austria, Finland, Netherlands, New Zealand, and Sweden), PCV10 serotypes contributed to only $2-14 \%$ of the remaining
PCV20-type disease burden (excluding nonPCV20 serotypes). Most of the remaining PCV20-type disease $(30-41 \%$ in Netherlands, New Zealand, and Sweden and 62-85\% in Austria and Finland) is attributable to PCV13unique serotypes, predominantly caused by serotypes 3 and 19A, which are contained in PCV13 but are left uncovered by PCV10. In countries using PCV10, serotype 19A has been observed to increase in both the proportion of disease and in the absolute number of cases [56]. For this reason, disease caused by PCV15and PCV20-unique serotypes make up a smaller proportion of remaining disease in these countries, corresponding to an additional $4-15 \%$ and 9-50\% of remaining PCV20-type disease, respectively. Both Sweden and New Zealand had lower proportions of PCV13-unique serotypes compared to the other PCV10 NIPs, potentially due to partial protection of PCV13 from its use in several counties in Sweden, and residual impact of the previous PCV13 NIP implemented in New Zealand from 2014 to 2017.

In countries using PCV13 as part of their current NIP at the time of data availability (i.e., Australia, Canada, France, Germany, Italy, South Korea, Spain, and UK), the remaining burden of PCV15- and PCV20-unique serotypes was higher than in countries using PCV10. In these countries with PCV13 NIPs, a smaller proportion of disease attributable to serotypes contained in currently licensed PCVs was identified, with a substantially greater proportion of disease preventable by investigational PCVs; notably, $2-33 \%$ of PCV20-type disease burden is due to PCV15-unique serotypes and $16-69 \%$ is due to PCV20-unique serotypes. Both investigational PCVs will provide substantially more protection than current PCVs, with PCV20 addressing a markedly greater disease burden compared to PCV13. Australia had the highest remaining burden of PCV13 serotypes among PCV13 NIPs, which may be due to the prior use of the $3+0$ schedule compared to other countries which utilize a booster dose in either a $2+1$ or $3+1$ schedule. As of July 2018, Australia has made the decision to move to a $2+1$ schedule, thus serotype distributions may change in future years [52]. 
This juxtaposition between the burden of disease in PCV13 and PCV10 NIPs is important for several reasons. First, countries that introduce a higher-valent PCV observe an analogous increase in the proportion of disease due to non-vaccine serotypes. In countries with PCV10 NIPs, this increase has largely been due to serotype 19A, given its high invasiveness and propensity for carriage, transmission, and antimicrobial resistance [56]. Meanwhile, in countries using PCV13, disease attributable to the incremental serotypes above those covered by PCV10 (i.e., PCV13-unique serotypes) has largely been reduced, and consequently the largest share of residual disease is due to nonPCV13 serotypes. Therefore, unlike PCV10 NIP countries, PCV13 NIP countries experience greater variability in the disease-causing serotypes. Importantly, while the serotype distribution has shifted, the overall incidence of disease has consistently declined over time with the introduction of higher-valent PCVs regardless of serotype replacement. In the UK, for example, incidence of IPD pre-PCV13 NIP (2008-2010) was 21.6 per 100,000 in children under 2 years of age, but fell to 13.9 per 100,000 in 2016/2017 despite the increase in nonPCV13 serotype disease (7.7 per 100,000 in $2008-2010$ vs. 12.3 per 100,000 in $2016 / 2017$ ) [11]. This serotype replacement phenomenon is likely due to an "unmasking" of non-PCV13 serotypes in carriage after widespread introduction of a PCV into pediatric NIPs, as less invasive circulating serotypes have the opportunity to cause disease, albeit at lower levels than prePCV13 [27].

Second, the burden of disease as estimated across the included countries in this analysis not only varies due to the choice of PCV in the NIP, but also by the population's PCV uptake, the duration of PCV use, dosing schedules, and host, pathogen, and environmental epidemiologic variability. Data availability also varies by country, with different years of data being used as model inputs that could impact interpretation and comparability of disease burden estimates across countries. Consequently, consideration of each of these factors is important for interpretation of PCV serotype coverage and disease burden estimates; thus, results should be interpreted individually for each country. In the subset of countries selected, most countries reported data at least 5 years after PCV NIP introduction, which typically corresponds to high population PCV uptake, and have utilized a $2+1$ dosing schedule. Therefore, aside from choice of PCV in the NIP, differences in PCV serotype coverage and disease burden estimates can also be explained by special NIP characteristics, such as differences in regional PCV NIPs or switching from PCV13 to PCV10 and vice versa. Specifically, each country has implemented the vaccine in different manners and at various times across the last decade. In Spain, Italy, and Canada, there is a national recommendation for PCV in children, but implementation is on a regional basis as compared to national implementation, and in Spain a PCV NIP was not introduced until 2015/2016 with low PCV13 uptake until recent years [62]. Additionally, in some countries where multiple vaccines are used in clinical practice (i.e., Canada, Germany, Italy, South Korea, and Sweden), there may be suboptimal overall protection of all vaccine serotypes at a national level.

Two recent studies by Hu et al. quantified the additional economic burden of PCV15 serotypes in the United States [63] and Europe [64] and found directionally similar results to our study; however, they did not include the burden of the five additional serotypes contained in PCV20. By omitting the incremental burden of PCV20-unique serotypes from their calculations, $\mathrm{Hu}$ et al. overestimates the burden of PCV15-unique serotypes and consequently misrepresents the vaccine-type burden of pneumococcal disease by only using PCV15type disease as a denominator. These studies also considered pre-PCV incidence of both PCV7 and PCV13-unique serotypes, while we only considered the most recent serotype coverage and burden of disease to characterize the remaining burden of vaccine-preventable pneumococcal disease rather than historical estimates. Although it is beneficial to show the overall value of a PCV program, this methodology does not reflect the current burden of disease which is perturbed by decades of PCV use. Estimating the current pneumococcal 
disease burden is important when policymakers must characterize which vaccines will provide robust serotype coverage and decrease the disease burden in their country, given that serotype epidemiology has changed over time and current disease rates are not analogous to prePCV incidence and serotype distributions. However, it remains essential that investigational higher-valent vaccines continue to protect against serotypes contained in currently licensed vaccines and avoid removing vaccine pressure, given that uncovered serotypes can rebound and cause significant disease. Such resurgence was observed in Belgium following the switch from PCV13 to PCV10 in their NIP, which resulted in a significant increase of serotype 19A disease [65-67].

Despite the significant disease burden estimated in this study, there are several limitations in this analysis that may contribute to an underestimation of the total vaccine-preventable pneumococcal disease. First, not all IPD cases are laboratory-confirmed (because a blood culture may not be sought or the patient may have already been treated with antibiotics), serotyped (because it may not be standard practice), or required to be reported to national surveillance, contributing to the likely underestimation of IPD burden. Additionally, there are variations in pneumococcal IPD serotype distributions across 0- to 5-year-olds; however, we present this evidence as a monolith due to inconsistently reported age strata across markets. Second, although real-world effectiveness studies have determined that PCV use has a larger impact on non-specific, all-cause PNE $[18,21,68]$ and $\mathrm{OM}[14,15,17]$ incidence, results conservatively estimated the pneumococcal-specific non-IPD burden. Moreover, the proportion of all-cause non-IPD due to pneumococcus is consistently underreported because of the lack of a robust diagnostic tool to identify the causative disease pathogen in children with PNE or OM [69, 70]. Many of the countries in our analysis conservatively assumed only $20 \%$ of PNE and OM were attributable to S. pneumoniae, despite observed reductions in $\mathrm{OM}$ and PNE in children as high as $41 \%$ [14] and $47 \%$ [18] after PCV13 introduction, respectively. This suggests that such a narrow definition of disease burden could be underestimating the potential impact of higher-valent PCVs in the future. Third, this study did not include vaccine-preventable disease burden across all age groups resulting from the extended benefits of infant vaccination among unvaccinated individuals. By providing direct protection to children under 5 years of age against serotypes contained in the vaccine, transmission of disease is disrupted through the reduction of nasopharyngeal carriage, and older populations also observe reduced pneumococcal disease incidence $[11,71,72]$. Therefore, the total vaccine-preventable disease burden due to infant vaccination could be significantly broader than the scope of this assessment. Fourth, this study only considered direct healthcare costs per disease event in calculating the economic burden attributable to incremental PCV serotypes. However, many other costs are attributable to these outcomes, including long-term sequalae costs, as well as indirect costs to society incurred from additional pneumococcal disease cases, such as the hours of lost productivity to patients, parents, and/or their caregivers, which when considered in the analysis would substantially increase the incremental economic burden averted by higher-valent PCVs.

Finally, an important limitation of this study is that the epidemiologic inputs included in the model were derived prior to the coronavirus disease 2019 (COVID-19) pandemic. Government lockdowns and the implementation of social distancing have been observed to drastically decrease the burden of respiratory illnesses along with pneumococcal disease. For example, in England, IPD incidence in the 2019/2020 epidemiologic year was $30 \%$ lower compared to $2018 / 2019$ across all ages, with significant reductions during the February to June 2020 lockdown period [73]. Further years of surveillance data will be needed to understand the full impact of government lockdown measures on the circulation of pneumococcal disease, both in terms of the incidence of overall disease, as well as potential changes in underlying serotype epidemiology. However, several models have indicated that, with relaxed social distancing and consequent disease circulation, the incidence of pneumococcal disease may return to 
pre-COVID levels by 2023-2024, suggesting that our results are indicative of the burden of disease at the onset of expected higher-valent PCV implementation [74, 75].

\section{CONCLUSION}

The clinical and economic burden of pneumococcal disease is largely driven by serotypes not covered by the PCV currently included in a country's NIP. Countries with PCV10 NIPs have a higher proportion of current vaccine-preventable disease burden than PCV13 countries, with disease primarily caused by serotypes contained in PCV13. Residual disease in countries with PCV13 NIPs is primarily attributable to non-PCV13 serotypes. Investigational PCVs with broader serotype coverage could reduce the clinical and economic burden of pneumococcal disease through breadth of coverage and by targeting serotypes that cause severe clinical outcomes. The incremental PCV20 serotypes contribute to a substantial proportion of annual pneumococcal disease cases, deaths, and direct health system costs that would not be prevented by current or other investigational lower-valent PCVs.

\section{ACKNOWLEDGEMENTS}

Funding. This work, including the Journal's Rapid Service Fee, was funded by Pfizer Inc.

\section{Medical Writing/Editorial Assis-} tance. Paranjoy Saharia and Shubhra Singh as part of IQVIA Real World Solutions provided medical writing and editorial assistance with funding received from Pfizer Inc.

Authorship. All named authors meet the International Committee of Medical Journal Editors (ICMJE) criteria for authorship for this article, take responsibility for the integrity of the work as a whole, and have given their approval for this version to be published.
Author Contributions. Matt D Wasserman, Johnna Perdrizet, and Raymond Farkouh conceived of the study, Matt D Wasserman, Emily K Horn, Johnna Perdrizet, Raymond A Farkouh, Kyla Hayford, and Lindsay Grant participated in its design and coordination, and Shubhra Singh, Paranjoy Saharia, Matt D Wasserman, and Emily Horn performed the analyses. All authors helped to draft the manuscript and read and approved the final manuscript.

Disclosures. Matt D Wasserman, Emily K Horn, Johnna Perdrizet, Raymond A Farkouh, Kyla Hayford, and Lindsay Grant are employees of Pfizer Inc. and may own stock or stock options. Shubhra Singh and Paranjoy Saharia received consulting fees from Pfizer Inc. for the study and manuscript development.

Compliance with Ethics Guidelines. This article is based on previously conducted studies and does not contain any new studies with human participants or animals performed by any of the authors.

Data Availability. All data generated or analyzed during this study are included in this published article/as supplementary information files.

Open Access. This article is licensed under a Creative Commons Attribution-NonCommercial 4.0 International License, which permits any non-commercial use, sharing, adaptation, distribution and reproduction in any medium or format, as long as you give appropriate credit to the original author(s) and the source, provide a link to the Creative Commons licence, and indicate if changes were made. The images or other third party material in this article are included in the article's Creative Commons licence, unless indicated otherwise in a credit line to the material. If material is not included in the article's Creative Commons licence and your intended use is not permitted by statutory regulation or exceeds the permitted use, you will need to obtain permission directly from the copyright holder. To view a copy of this licence, visit http://creativecommons.org/licenses/by$\mathrm{nc} / 4.0 /$. 


\section{REFERENCES}

1. Chapman R, Sutton K, Dillon-Murphy D, Patel S, Hilton B, Farkouh R, et al. Ten year public health impact of 13-valent pneumococcal conjugate vaccination in infants: a modelling analysis. Vaccine. 2020;38(45):7138-45.

2. Wahl B, O'Brien KL, Greenbaum A, Majumder A, Liu L, Chu Y, et al. Burden of Streptococcus pneumoniae and Haemophilus influenzae type $\mathrm{b}$ disease in children in the era of conjugate vaccines: global, regional, and national estimates for 2000-15. Lancet Glob Health. 2018;6(7):e744-57.

3. Henriques-Normark B, Tuomanen EI. The pneumococcus: epidemiology, microbiology, and pathogenesis. Cold Spring Harb Perspect Med. 2013;3(7): a010215.

4. GBD 2016 Lower Respiratory Infections Collaborators. Estimates of the global, regional, and national morbidity, mortality, and aetiologies of lower respiratory infections in 195 countries, 1990-2016: a systematic analysis for the Global Burden of Disease Study 2016. Lancet Infect Dis. 2016;18(11): 1191-210.

5. Ganaie F, Saad JS, McGee L, van Tonder AJ, Bentley SD, Lo SW, et al. A new pneumococcal capsule type, $10 \mathrm{D}$, is the 100th serotype and has a large cps fragment from an oral streptococcus. MBio. 2020. https://doi.org/10.1128/mBio.00937-20.

6. Tin Tin Htar M, Christopoulou D, Schmitt H-J. Pneumococcal serotype evolution in Western Europe. BMC Infect Dis. 2015;15(1):419.

7. De Carvalho Gomes H, Muscat M, Monnet DL, Giesecke J, Lopalco PL. Use of seven-valent pneumococcal conjugate vaccine (PCV7) in Europe, 2001-2007. Euro Surveill. 2009. https://doi.org/10. 2807/ese.14.12.19159-en.

8. Berical AC, Harris D, Dela Cruz CS, Possick JD. Pneumococcal vaccination strategies. An update and perspective. Ann Am Thorac Soc. 2016;13(6): 933-44.

9. Horn EK, Wasserman MD, Hall-Murray C, Sings HL, Chapman R, Farkouh RA. Public health impact of pneumococcal conjugate vaccination: a review of measurement challenges. Expert Rev Vaccines. 2021. https://doi.org/10.1080/14760584.2021. 1971521.

10. Moore MR, Link-Gelles R, Schaffner W, Lynfield R, Lexau C, Bennett NM, et al. Effect of use of 13-valent pneumococcal conjugate vaccine in children on invasive pneumococcal disease in children and adults in the USA: analysis of multisite, population- based surveillance. Lancet Infect Dis. 2015;15(3): 301-9.

11. Ladhani SN, Collins S, Djennad A, Sheppard CL, Borrow R, Fry NK, et al. Rapid increase in nonvaccine serotypes causing invasive pneumococcal disease in England and Wales, 2000-17: a prospective national observational cohort study. Lancet Infect Dis. 2018;18(4):441-51.

12. Lepoutre A, Varon E, Georges S, Dorléans F, Janoir C, Gutmann L, et al. Impact of the pneumococcal conjugate vaccines on invasive pneumococcal disease in France, 2001-2012. Vaccine. 2015;33(2): 359-66.

13. Harboe ZB, Thomsen RW, Riis A, Valentiner-Branth $\mathrm{P}$, Christensen JJ, Lambertsen L, et al. Pneumococcal serotypes and mortality following invasive pneumococcal disease: a population-based cohort study. PLoS Med. 2009;6(5): e1000081.

14. Marom T, Tan A, Wilkinson GS, Pierson KS, Freeman JL, Chonmaitree T. Trends in otitis media-related health care use in the United States, 2001-2011. JAMA Pediatr. 2014;168(1):68-75.

15. Dagan R, van der Beek BA, Ben-Shimol S, Pilishvili $\mathrm{T}$, Givon-Lavi N. Effectiveness of the seven- and thirteen valent pneumococcal conjugate vaccines against vaccine-serotype otitis media. Clin Infect Dis. 2021. https://doi.org/10.1093/cid/ciab066.

16. Ben-Shimol S, Givon-Lavi N, Leibovitz E, Raiz S, Greenberg D, Dagan R. Near-elimination of otitis media caused by 13 -valent pneumococcal conjugate vaccine (PCV) serotypes in southern Israel shortly after sequential introduction of 7-valent/13valent PCV. Clin Infect Dis. 2014;59(12):1724-32.

17. Lau WCY, Murray M, El-Turki A, Saxena S, Ladhani $\mathrm{S}$, Long $\mathrm{P}$, et al. Impact of pneumococcal conjugate vaccines on childhood otitis media in the United Kingdom. Vaccine. 2015;33(39):5072-9.

18. Greenberg D, Givon-Lavi N, Ben-Shimol S, Ziv JB, Dagan R. Impact of PCV7/PCV13 introduction on community-acquired alveolar pneumonia in children $<5$ years. Vaccine. 2015;33(36):4623-9.

19. Griffin MR, Zhu Y, Moore MR, Whitney CG, Grijalva CGUS. hospitalizations for pneumonia after a decade of pneumococcal vaccination. N Engl J Med. 2013;369(2):155-63.

20. Becker-Dreps S, Amaya E, Liu L, Moreno G, Rocha J, Briceño $\mathrm{R}$, et al. Changes in childhood pneumonia and infant mortality rates following introduction of the 13-valent pneumococcal conjugate vaccine in Nicaragua. Pediatr Infect Dis J. 2014;33(6):637-42. 
21. Angoulvant F, Levy C, Grimprel E, Varon E, Lorrot M, Biscardi S, et al. Early impact of 13-valent pneumococcal conjugate vaccine on communityacquired pneumonia in children. Clin Infect Dis. 2014;58(7):918-24.

22. van Hoek AJ, Sheppard CL, Andrews NJ, Waight PA, Slack MPE, Harrison TG, et al. Pneumococcal carriage in children and adults two years after introduction of the thirteen valent pneumococcal conjugate vaccine in England. Vaccine. 2014;32(34):4349-55.

23. Rodgers GL, Klugman KP. Surveillance of the impact of pneumococcal conjugate vaccines in developing countries. Hum Vaccin Immunother. 2016;12(2):417-20.

24. Chen C, Cervero Liceras F, Flasche S, Sidharta S, Yoong J, Sundaram N, et al. Effect and cost-effectiveness of pneumococcal conjugate vaccination: a global modelling analysis. Lancet Glob Health. 2019;7(1):e58-67.

25. Wasserman M, Sings HL, Jones D, Pugh S, Moffatt M, Farkouh R. Review of vaccine effectiveness assumptions used in economic evaluations of infant pneumococcal conjugate vaccine. Expert Rev Vaccines. 2018;17(1):71-8.

26. Balsells E, Dagan R, Yildirim I, Gounder PP, Steens A, Muñoz-Almagro $C$, et al. The relative invasive disease potential of Streptococcus pneumoniae among children after PCV introduction: a systematic review and meta-analysis. J Infect. 2018;77(5): 368-78.

27. Løchen A, Croucher NJ, Anderson RM. Divergent serotype replacement trends and increasing diversity in pneumococcal disease in high income settings reduce the benefit of expanding vaccine valency. Sci Rep. 2020;10(1):18977.

28. Savulescu C, Krizova P, Lepoutre A, Mereckiene J, Vestrheim DF, Ciruela P, et al. Effect of high-valency pneumococcal conjugate vaccines on invasive pneumococcal disease in children in SpIDnet countries: an observational multicentre study. Lancet Respir Med. 2017;5(8):648-56.

29. Dion SB, Major M, Gabriela Grajales A, Nepal RM, Cane A, Gessner B, et al. Invasive pneumococcal disease in Canada 2010-2017: The role of current and next-generation higher-valent pneumococcal conjugate vaccines. Vaccine. 2021;39(22):3007-17.

30. Balsells E, Guillot L, Nair H, Kyaw MH. Serotype distribution of Streptococcus pneumoniae causing invasive disease in children in the post-PCV era: a systematic review and meta-analysis. PLoS ONE. 2017;12(5): e0177113.
31. Levy C, Ouldali N, Caeymaex L, Angoulvant F, Varon E, Cohen R. Diversity of serotype replacement after pneumococcal conjugate vaccine implementation in Europe. J Pediatr. 2019;213:252253.e3.

32. Lo SW, Gladstone RA, van Tonder AJ, Lees JA, du Plessis M, Benisty R, et al. Pneumococcal lineages associated with serotype replacement and antibiotic resistance in childhood invasive pneumococcal disease in the post-PCV13 era: an international whole-genome sequencing study. Lancet Infect Dis. 2019;19(7):759-69.

33. Tomczyk S, Lynfield R, Schaffner W, Reingold A, Miller L, Petit S, et al. Prevention of antibioticnonsusceptible invasive pneumococcal disease with the 13-Valent pneumococcal conjugate vaccine. Clin Infect Dis. 2016;62(9):1119-25.

34. Cohen R, Biscardi S, Levy C. The multifaceted impact of pneumococcal conjugate vaccine implementation in children in France between 2001 to 2014. Hum Vaccin Immunother. 2016;12(2): 277-84.

35. Hausdorff WP, Hanage WP. Interim results of an ecological experiment - Conjugate vaccination against the pneumococcus and serotype replacement. Hum Vaccin Immunother. 2016;12(2): 358-74.

36. van Hoek AJ, Andrews N, Waight PA, George R, Miller E. Effect of serotype on focus and mortality of invasive pneumococcal disease: coverage of different vaccines and insight into non-vaccine serotypes. PLoS ONE. 2012;7(7): e39150.

37. Hausdorff WP, Feikin DR, Klugman KP. Epidemiological differences among pneumococcal serotypes. Lancet Infect Dis. 2005;5(2):83-93.

38. Richter SS, Diekema DJ, Heilmann KP, Dohrn CL, Riahi F, Doern GV. Changes in pneumococcal serotypes and antimicrobial resistance after introduction of the 13-valent conjugate vaccine in the United States. Antimicrob Agents Chemother. 2014;58(11):6484-9.

39. Varon E, Cohen R, Béchet S, Doit C, Levy C. Invasive disease potential of pneumococci before and after the 13-valent pneumococcal conjugate vaccine implementation in children. Vaccine. 2015;33(46):6178-85.

40. Ikuse T, Habuka R, Wakamatsu Y, Nakajima T, Saitoh N, Yoshida H, et al. Local outbreak of Streptococcus pneumoniae serotype $12 \mathrm{~F}$ caused high morbidity and mortality among children and adults. Epidemiol Infect. 2018;146(14):1793-6. 
41. Kaplan SL, Center KJ, Barson WJ, Ling-Lin P, Romero JR, Bradley JS, et al. Multicenter surveillance of Streptococcus pneumoniae isolates from middle ear and mastoid cultures in the 13-valent pneumococcal conjugate vaccine era. Clin Infect Dis. 2015;60(9):1339-45.

42. PREVNAR 20 $0^{\mathrm{TM}}$ Press Release. Pfizer.com. 2021 [cited $2021 \mathrm{Jul} 4$ ]. Available from: https://www. pfizer.com/news/press-release/press-release-detail/ us-fda-approves-prevnar-20tm-pfizerspneumococcal-20-valent

43. VAXNEUVANCE ${ }^{\mathrm{TM}}$ Press Release. Merck.com. 2021 [cited 2021 Aug 4]. Available from: https://www. merck.com/news/merck-announces-u-s-fdaapproval-of-vaxneuvance-pneumococcal-15-valentconjugate-vaccine-for-the-prevention-of-invasivepneumococcal-disease-in-adults-18-years-andolder-caused-by-15-serot/

44. Impfplan Österreich. Sozialministerium.at. [cited 2021 Apr 29]. Available from: https://www. sozialministerium.at/Themen/Gesundheit/Impfen/ Impfplan-\%C3\%96sterreich.html

45. RKI - Archiv 2015 - Wissenschaftliche Begründung der STIKO zur Änderung der PneumokokkenImpfempfehlung für Säuglinge. Rki.de. [cited 2021 Jul 29]. Available from: https://www.rki.de/DE/ Content/Infekt/EpidBull/Archiv/2015/36/Art_01. html

46. Kim H-Y, Park S-B, Kang E-S, Lee S-M, Kim H-J, Wasserman M. Cost-effectiveness of a national immunization program with the 13-valent pneumococcal conjugate vaccine compared with the 10 -valent pneumococcal conjugate vaccine in South Korea. Hum Vaccin Immunother. 2021;17(3):909-18.

47. Naucler P, Galanis I, Morfeldt E, Darenberg J, Örtqvist $\AA$, Henriques-Normark B. Comparison of the impact of pneumococcal conjugate vaccine 10 or pneumococcal conjugate vaccine 13 on invasive pneumococcal disease in equivalent populations. Clin Infect Dis. 2017;65(11):1780-9.

48. View-Hub by IVAC. Vaccine product (current/planned). View-hub.org. [cited 2021 Apr 29]. Available from: https://view-hub.org/map/?set= current-vaccine-intro-status\&group=vaccineintroduction\& $\&$ ategory $=\mathrm{pcv}$

49. Miller E, Andrews NJ, Waight PA, Slack MP, George RC. Herd immunity and serotype replacement 4 years after seven-valent pneumococcal conjugate vaccination in England and Wales: an observational cohort study. Lancet Infect Dis. 2011;11(10):760-8.

50. Melegaro A, Edmunds WJ. Cost-effectiveness analysis of pneumococcal conjugate vaccination in
England and Wales. Vaccine. 2004;22(31-32): 4203-14.

51. Wilson $M$, Wasserman $M$, Jadavi $T$, Postma $M$, Breton M-C, Peloquin F, et al. Clinical and economic impact of a potential switch from 13-Valent to 10 -Valent pneumococcal conjugate infant vaccination in Canada. Infect Dis Ther. 2018;7(3): 353-71.

52. Perdrizet J, Lai YS, Williams S, Struwig VA, Wasserman M. Retrospective impact analysis and cost-effectiveness of the pneumococcal conjugate vaccine infant program in Australia. Infect Dis Ther. 2021;10(1):507-20.

53. Wasserman M, Palacios MG, Grajales AG, Baez Revueltas FB, Wilson M, McDade C, et al. Modeling the sustained use of the 13-valent pneumococcal conjugate vaccine compared to switching to the 10 -valent vaccine in Mexico. Hum Vaccin Immunother. 2019;15(3):560-9.

54. Cooper D, Yu X, Sidhu M, Nahm MH, Fernsten P, Jansen KU. The 13-valent pneumococcal conjugate vaccine (PCV13) elicits cross-functional opsonophagocytic killing responses in humans to Streptococcus pneumoniae serotypes 6C and 7A. Vaccine. 2011;29(41):7207-11.

55. Spencer BL, Shenoy AT, Orihuela CJ, Nahm MH. The pneumococcal serotype $15 \mathrm{C}$ capsule is partially O-acetylated and allows for limited evasion of 23-Valent pneumococcal polysaccharide vaccineelicited anti-serotype 15B antibodies. Clin Vaccine Immunol. 2017. https://doi.org/10.1128/cvi.0009917.

56. Isturiz R, Sings HL, Hilton B, Arguedas A, Reinert R-R, Jodar L. Streptococcus pneumoniae serotype 19A: worldwide epidemiology. Expert Rev Vaccines. 2017;16(10):1007-27.

57. Garcia Quesada M, Yang Y, Bennett JC, Hayford K, Zeger SL, Feikin DR, et al. Serotype distribution of remaining pneumococcal meningitis in the mature PCV10/13 period: findings from the PSERENADE project. Microorganisms. 2021;9(4):738.

58. Waight PA, Andrews NJ, Ladhani SN, Sheppard CL, Slack MPE, Miller E. Effect of the 13-valent pneumococcal conjugate vaccine on invasive pneumococcal disease in England and Wales 4 years after its introduction: an observational cohort study. Lancet Infect Dis. 2015;15(5):535-43.

59. Moore CE, Paul J, Foster D, Mahar SA, Griffiths D, Knox K, et al. Reduction of invasive pneumococcal disease 3 years after the introduction of the 13-valent conjugate vaccine in the Oxfordshire region of England. J Infect Dis. 2014;210(7):1001-11. 
60. Marchisio P, Esposito S, Picca M, Baggi E, Terranova L, Orenti A, et al. Serotypes not included in 13-Valent pneumococcal vaccine as causes of acute otitis media with spontaneous tympanic membrane perforation in a geographic area with high vaccination coverage. Pediatr Infect Dis J. 2017;36(5): 521-3.

61. Morales M, Ludwig G, Ercibengoa M, Esteva C, Sanchez-Encinales V, Alonso M, et al. Changes in the serotype distribution of Streptococcus pneumoniae causing otitis media after PCV13 introduction in Spain. PLoS ONE. 2018;13(12): e0209048.

62. Moreira M, Castro O, Palmieri M, Efklidou S, Castagna $\mathrm{S}$, Hoet B. A reflection on invasive pneumococcal disease and pneumococcal conjugate vaccination coverage in children in Southern Europe (2009-2016). Hum Vaccin Immunother. 2017;13(6):1-12.

63. Hu T, Weiss T, Owusu-Edusei K, Petigara T. Health and economic burden associated with 15-valent pneumococcal conjugate vaccine serotypes in children in the United States. J Med Econ. 2020;23(12): 1653-60.

64. Hu T, Weiss T, Bencina G, Owusu-Edusei K, Petigara $\mathrm{T}$. Health and economic burden of invasive pneumococcal disease associated with 15-valent pneumococcal conjugate vaccine serotypes in children across eight European countries. J Med Econ. 2021;24(1):1098-107.

65. Desmet S, Lagrou K, Wyndham-Thomas C, Braeye $\mathrm{T}$, Verhaegen J, Maes P, et al. Dynamic changes in paediatric invasive pneumococcal disease after sequential switches of conjugate vaccine in Belgium: a national retrospective observational study. Lancet Infect Dis. 2021;21(1):127-36.

66. Wouters I, Desmet S, Van Heirstraeten L, Herzog SA, Beutels P, Verhaegen J, et al. How nasopharyngeal pneumococcal carriage evolved during and after a PCV13-to-PCV10 vaccination programme switch in Belgium, 2016 to 2018. Euro Surveill. 2020. https:// doi.org/10.2807/1560-7917.ES.2020.25.5.1900303.

67. Wilson MR, McDade CL, Perdrizet JE, Mignon A, Farkouh RA, Wasserman MD. Validation of a novel forecasting method for estimating the impact of switching pneumococcal conjugate programs: Evidence from Belgium. Infect Dis Ther. 2021. https:// doi.org/10.1007/s40121-021-00485-9.

68. Griffin MR, Mitchel E, Moore MR, Whitney CG, Grijalva CG, Centers for Disease Control and
Prevention (CDC). Declines in pneumonia hospitalizations of children aged $<2$ years associated with the use of pneumococcal conjugate vaccines Tennessee, 1998-2012. Morb Mortal Wkly Rep. 2014;63(44):995-8.

69. Shea KM, Edelsberg J, Weycker D, Farkouh RA, Strutton DR, Pelton SI. Rates of pneumococcal disease in adults with chronic medical conditions. Open Forum Infect Dis. 2014;1(1): ofu024.

70. Pelton SI, Shea KM, Farkouh RA, Strutton DR, Braun $\mathrm{S}$, Jacob C, et al. Rates of pneumonia among children and adults with chronic medical conditions in Germany. BMC Infect Dis. 2015;15(1):470.

71. Shiri T, Datta S, Madan J, Tsertsvadze A, Royle P, Keeling MJ, et al. Indirect effects of childhood pneumococcal conjugate vaccination on invasive pneumococcal disease: a systematic review and meta-analysis. Lancet Glob Health. 2017;5(1): e51-9.

72. Ahmed SS, Pondo T, Xing W, McGee L, Farley M, Schaffner W, et al. Early impact of 13-Valent pneumococcal conjugate vaccine use on invasive pneumococcal disease among adults with and without underlying medical conditions-United States. Clin Infect Dis. 2020;70(12):2484-92.

73. Amin-Chowdhury Z, Aiano F, Mensah A, Sheppard CL, Litt D, Fry NK, et al. Impact of the Coronavirus disease 2019 (COVID-19) pandemic on invasive pneumococcal disease and risk of pneumococcal coinfection with severe acute respiratory syndrome Coronavirus 2 (SARS-CoV-2): Prospective national cohort study. England Clin Infect Dis. 2021;72(5): e65-75.

74. Choi YH, Miller E. Potential impact of Covid-19 response measures on invasive pneumococcal disease in England and Wales. bioRxiv. 2020. https:// doi.org/10.1101/2020.06.01.20119057.

75. Kitano T, Aoki H. A model for the incremental burden of invasive Haemophilus influenzae type $\mathrm{b}$ due to a decline of childhood vaccination during the COVID-19 outbreak: a dynamic transmission model in Japan. Vaccine. 2021;39(2):343-9.

\section{Publisher's Note}

Springer Nature remains neutral with regard to jurisdictional claims in published maps and institutional affiliations. 